\title{
ESTADO, MERCADO, SECTOR PRIVADO Y PLANIFICACIÓN EN EL DESARROLLO ECONÓMICO RECIENTE DE CHINA
}

Elias Jabbour ${ }^{1}$

Alexis Dantas ${ }^{2}$

Resumen: El objetivo de este artículo es demostrar, partiendo de una visión de conjunto de las reformas económicas chinas, que el surgimiento de un amplio sector privado y la propia sofisticación y diversificación de la manufactura ha demandado continua reorganización de actividades entre los sectores estatal y privado de la economía. En este artículo sostenemos que el Estado pasó a contener mayor protagonismo en las esferas que involucran el control de la gran industria y de las grandes finanzas, bien como al nivel de la coordinación y socialización de la inversión por la vía del comando sobre las políticas económica, monetaria y fiscal, del comercio exterior y, principalmente, al lanzamiento de nuevas y superiores formas de planificación económica.

Palabras claves: China; Reformas económicas; Estado; Sector privado; Planificación económica.

\section{ESTADO, MERCADO, SETOR PRIVADO E PLANIFICAÇÃO NO DESENVOLVIMENTO ECONÔMICO RECENTE DA CHINA}

Resumo: O objetivo deste artigo é demonstrar, partindo de uma visão de conjunto das reformas econômicas chinesas, que o surgimento de um largo setor privado e a própria sofisticação e diversificação da manufatura têm demandado contínua reorganização de atividades entre os setores estatal e privado da economia. Neste artigo sustentamos que o Estado passou a caber maior protagonismo nas esferas que envolvem o controle da grande indústria e da grande finança, assim como no nível da coordenação e socialização do investimento pela via do comando sobre as políticas econômica, monetária e fiscal, do comercio exterior e, principalmente, no lançamento de novas e superiores formas de planificação econômica.

Palavras-chave: China; Reformas econômicas; Estado; Setor privado; Planejamento econômico

\section{STATE, MARKET, PRIVATE SECTOR IN RECENT CHINA'S ECONOMIC DEVELOPMENT}

Abstract: The main of this article is to demonstrate, from an over view of China's economic reforms, that the emergence of a large private sector and the sophistication and diversification of the industry has required continued reorganization activities between the state and private sector sof the economy. We support in this paper the state began to develop major role in the spheres involving the control of the major industry sector sand big finance, as well as the coordination and socialization of investment - in this case,

\footnotetext{
${ }^{1}$ Profesor Adjunto de la Facultad de Ciencias Económicas de la Universidad del Estado de Río de Janeiro (FCE-UERJ). Email: eliasjabbour@terra.com.br

${ }^{2}$ Profesor Asociado de la Facultad de Ciencias Económicas de la Universidad del Estado de Río de Janeiro (FCE-UERJ). Email: alexis.dantas@gmail.com
} 
economic policy (monetary and fiscal), foreign trade and especially, the laun chof innovative and superior form sof economic planning.

Keywords: China; Economic reforms; State; Private sector; Economic planning

\section{INTRODUCCIÓN}

En una trayectoria de semejanza con la estadounidense entre la segunda mitad del siglo XIX (unificación territorial) y el final de la Segunda Guerra Mundial, el crecimiento económico robusto, continuo y el consecuente aumento de influencia de la República Popular de China en el mundo contemporáneo tal vez sea el hecho, político y económico, más relevante de la época en que vivimos.

Al circunscribir el análisis sólamente a los años post reformas $(1978)^{3}$, la tasa media del crecimiento del Producto Interno Bruto (PIB) se aproxima de los $10 \%$ anuales con renta per cápita (por Paridad de Poder de Compra), saliendo de US\$ 250 dólares americanos en 1980 para US\$ 9.040 en 2014. Desde 2013, China es el país de mayor volumen de comercio exterior, provocando efectos de demanda y de estructura sobre prácticamente todas las economías nacionales, volviéndose -también- gran exportador de capitales con Inversiones Extranjeras Directas (IED's), pasando de US\$ 0,8 mil millones en 1990 para US\$ 140 mil millones en 2014 . Como receptores, los IED's salieron de US\$ 1,4 mil millones en 1984 para US\$ 119,6 mil millones em 2014. Retornando a la variable comercio exterior, el peso de su influencia internacional y la percepción de una combinación entre un modelo export-led con rasgos de mercantilismo moderno puede ser notado en la evolución de esta pauta. En 1978, las exportaciones/importaciones fueron del orden de US\$ 9,75 mil millones/US\$10,89 mil millones, respectivamente. En 2014, los mismos agregados alcanzaron US\$ 2,34 billones/US\$1,96 billón. La formación de las mayores reservas cambiales del mundo

\footnotetext{
${ }^{3}$ El desarrollismo chino no puede ser observado como una tendencia inaugurada con las reformas económicas de 1978. En este contexto, es más prudente tipificar las reformas económicas como el proceso de internación del desarrollismo de tipo asiático en sustitución a una dinámica de crecimiento, históricamente determinado, que penalizaba el campo en beneficio de la ciudad, evitando así la simple negación del período anterior, tan perjudicial al análisis de fondo sobre procesos históricos complejos. Cabe señalar que entre 1953 y 1978, la tasa media de crecimento del PIB fue del 6,6\% al año, detrás apenas de sus vecinos del Sudeste Asiático. En este período se sentaron las bases para consecución de grandes proyectos hidráulicos (Ertan), la confección de la bomba nuclear (1964), el lanzamiento de satélites artificiales (1971) y la construcción del metro de Pekín (1975) dotado de equipos exclusivamente chinos. Ignacio Rangel, en desconocido artículo escrito en 1952, ya apuntaba a la confirmada tendencia de que China contía la paradoja de un país agrícola, pero exportador de productos manufacturados. Sobre este tema, léase: Rangel (1952).

${ }^{4}$ América Latina es un caso de combinación entre efectos de estructura y demanda con la elevación de la participación china en sus pautas exportadoras e importadoras. Sobre este tema, léase: Medeiros e Cintra (2015).
} 
obedeció a la misma lógica cuantitativa del comercio externo, saltando de US\$ 1,6 mil millón en 1978 para US\$ 3,84 billones en diciembre de 2014 (NBSC, 2015). Entre las TOP 500 de la revista Forbes, están presentes 43 empresas chinas, 20 de las cuales conglomerados estatales están ubicados en las primeras 250 posiciones del ranking de $2013^{5}$.

El mantenimiento de altas tasas de inversión y, consecuentemente, un aumento "en el frente" de capacidad productiva instalada sumadas al alcance de un moderno sistema financiero garantizó al país la construcción de "diques contra mareas históricas desfavorables" (Kissinger, 2011, p.446), como se ha percibido en la resistencia a los primeros años de la crisis financiera internacional por la vía de un programa de inversiones en infraestructuras del orden de US\$ 600 mil millones. Curiosamente, la extensión de la dinámica de acumulación centrada en la inversión, como recurso a la resistencia a la crisis internacional, fue motivo de mayor agotamiento de la propia dinámica y la consecuente urgencia de transición a un patrón centrado en la movilización de recursos para fines de consumo. He ahí un rasgo en forma de desequilibrio estructural que se intercala con una coyuntura interna de formación de burbujas financieras y alto nivel de endeudamiento provincial - preanuncios de una crisis que, antes de ser financiera, es expresión de un largo proceso interno de transición.

El desarrollo reciente chino es historia de grandes saltos, de un desequilibrio a otro. Con esa suposición, objetivamos con este artículo demostrar que el surgimiento de un amplio sector privado y la propia sofisticación y diversificación de la manufactura demandó una reorganización de actividades entre los sectores estatal y privado de la economía 6 . Al Estado pasó a pugnar mayor protagonismo en las esferas de las políticas económica, monetaria y fiscal, en el ámbito del comercio exterior y, sobre todo, al lanzamiento de nuevas y superiores formas de planificación económica (Jabbour, 2012) adyacentes a la gestión de macizas y rápidas intervenciones sobre el territorio y margen de maniobra al giro de compás del externo al interno en momentos de relativo cierre de mercados externos.

\footnotetext{
5"The World's Largest Companies: China Takes Over The Top Three Stops". Forbes. 07/05/2014. Disponible por el enlace: <http://www.forbes.com/global2000/list/\#tab:overall>.

${ }^{6}$ La percepción sobre la necesidad de reorganización de actividades entre el Estado y la iniciativa privada en concomitancia con determinados niveles de desarrollo de un país es muy presente en la obra de Ignacio Rangel con consecuencias virtuosas en las elaboraciones de Bresser Pereira sobre el tema. A nuestro ver, esta reorganización dinámica de actividades funciona como una ley objetiva del proceso de desarrollo a ser observada bajo el riesgo de la dogmatización y de la adopción de formas rígidas e ideologizadas de bordaje. Sobre este tema, léanse: Rangel (1981) y Bresser Pereira (1989).
} 
Además de esta introducción, este artículo está dividido en otras tres secciones. La segunda sección discutirá la Economía Política de las reformas con el objetivo de caracterizar el patrón de acumulación inaugurado con las reformas económicas en 1978, el papel del innercatch up (potencial de acumulación encerrado en los campesinos) anterior a la internación del modelo pautado por la combinación entre exportled y las altas tasas de inversión. Además de la (re)construcción del pacto de poder esencial al progreso de las reformas económicas expresada en la subversión del modelo soviético de industrialización por el privilegio a la agricultura y a los campesinos en relación a las ciudades, el consiguiente reordenamiento de las empresas rurales a la estrategia de conexión de mercados regionales e incluso la inserción internacional china. La tercera sección analiza la macrodinámica del proceso de las reformas con cotejo de datos y dinámica macroeconómica capaces de evidenciar el proceso de mutación del centro de gravedad, en la década de 1990, de una industrialización presionada por nuevos hábitos de consumo a la configuración de un centrum inversor y exportador. En la tercera sección trataremos, también, de la transformación del desarrollo económico en desarrollo territorial como expresión de la estrategia nacional definida, además del papel del propio Estado y de la gran finanza nacional en el proceso. En la conclusión, buscaremos sintetizar puntos importantes de nuestro análisis, además de dar mayor contorno a los principales desafíos de la gobernanza china en la actualidad.

\section{EL IMPULSO PRIMARIO}

La inauguración de las reformas económicas en 1978 coincide con una serie de eventos cuyo sentido tiene expresión en una nueva división internacional del trabajo inaugurada desde entonces. La pérdida de espacio económico y político estadounidense (ascensión económica alemana y japonesa, derrota militar en el Vietnam y creciente pérdida de influencia en el Tercer Mundo) había entablado una contraofensiva a escala internacional. Factor determinante para la realización de las reformas económicas residía, también, en la percepción de atraso del país en relación a los Estados desarrollistas de su alredor, incluyendo las dos grandes pendencias históricas de China post-1949, Hong Kong y Taiwán ${ }^{7}$, y el propio surgimiento de un nuevo paradigma tecnológico de su rival

\footnotetext{
${ }^{7}$ En 1978, Taiwán poseía una renta per capita 19,7 veces mayor que la verificada en la China continental (Jabbour, 2006, p.57), lo que hacía insostenible cualquier intento político hacia la reunificación del país.
} 
histórico, el Japón. En el campo del socialismo realmente existente es irresistible interconectar la decadencia del fordismo con la propia pérdida de dinamismo económico de la Unión Soviética y de sus socios del Consejo de Asistencia Económica Mutua (COMECON), algunos de los cuales atraídos a la propia órbita de la fase b del Ciclo Largo $^{8}$ inaugurado em 1973 con cataclismos políticos amplificados por la crisis de la deuda mexicana em 1982, especialmente los casos de Polonia y Iugoslavia.

Desde el punto de vista de la gran política interna, el principal factor a la modernización residió en la recomposición del pacto de poder inaugurado em 1949, pero prácticamente desmantelado en razón de la lógica de la llamada acumulación primitiva socialista y del radicalismo antimercantil anexas a las campañas como El Gran Salto Adelante (1956-1961) y la Revolución Cultural (1966-1976) ${ }^{9}$. Deng Xiaoping percibió, con sagacidad, que las mismas fuerzas (campesinas), que llevaron al Partido Comunista de China (PCCh) al poder en 1949, serían el motor de arranque de sus reformas económicas concentradas, en principio, en el restablecimiento de un sistema interno de abastecimiento alimentario capaz de superar la era de la escasez en la producción de alimentos al tiempo que garantizaba condiciones mínimas al surgimiento de un amplio mercado interno al consumo de bienes manufacturados. La política, la estrategia y la economía deberían fundirse, inicialmente, en una reverssión de la lógica de relaciones campo-ciudad intrínsecas al modelo soviético, desfavorables a la agricultura ${ }^{10}$. Los patrones clásicos de la división social del trabajo urgían la instalación en un proceso, de inicio, caracterizado por una liberalización sin privatización.

Por la lógica de la gran estrategia y del peso político de las zonas rurales, las reformas económicas buscaron su primer intento con la implantación de los contratos de responsabilidad entre las familias campesinas y el Estado, en una institución de cuotas de producción a ser entregadas - por las familias- al Estado, seguida del permiso a la comercialización del excedente producido ${ }^{11}$. Como resultado de esta política, en 1984 la

\footnotetext{
${ }^{8}$ Nos referimos a la Fase b del Ciclo Largo de Kondratief.

${ }^{9}$ El pacto de poder que llevó al PCCh al poder en 1949 tenía en los campesinos, amplia mayoría de la población, la principal base apoyo.

${ }^{10}$ Sobre el modelo soviético de desarrollo/acumulación primitiva socialista, léase: Domar (1972).

${ }^{11}$ No es inusual asociar los contratos de responsabilidad instituidos en China post-reformas con los mecanismos de acumulación insertados en la corta experiencia rusa de la Nueva Política Económica (NEP), propuesta por Lenin en 1921. Domenico Losurdo (2004, p.57) llega a clasificar las propias reformas chinas como una "gran NEP". En nuestra opinión, la existencia de recursos ociosos es un prerrequisito básico para el desarrollo, en que en una sociedad agraria la propia agricultura es fuente de recursos ociosos cuya plena utilización demanda cambios institucionales como punto de partida al proceso de acumulación, alcanzando, cualitativamente, la economía como un todo. Este es el caso de China en los estertores de las reformas económicas. Sobre la NEP, léase: Lenin (1921 [1964]).
} 
producción de cereales alcanzó el récord de 407,3 millones de toneladas con un aumento de 33,6\% en relación a la cosecha de 1978 (Rong et al., 1992, p.375). En este sentido, la caracterización de los primeiros años de reformas económicas en China, tal vez un ejemplo de "crecimiento fora del plan" (Naughton, 1996), es expresión de la recomposición de la pequeña producción mercantil en una especie de laissez faire campesino, generando desarrollo económico en relación de causa y consecuencia de la expansión de la economía de mercado. Expansión que no ocorrió solamente en beneficio de las estáticas y rústicas formas de planificación características del complejo agroindustrial de las comunas instituidas en la era Mao Tsétung, sino también de la pressión ejercida por esa nueva economía de mercado sobre la economía natural con efectos expansivos a la propia economía de mercado.

La aceleración del sector primario de la economía en los primeros años de las reformas fue um hecho con repercusiones políticas, y estratégicas, poco percibidas. Difícil no relacionar el amplio respaldo de las bases rurales al régimen con el mantenimiento y legitimación del PCCh durante el proceso de disolución de la Unión Soviética, del final de las experiencias del Este Europeo y las agitaciones que sacudieron Pekín entre mayo e junio de 1989. Las reformas rurales crearon un contorno político, hasta el momento, incontestable al proyecto estratégico insertado en las Cuatro Modernizaciones (agricultura, industria, ciencia y tecnología y defensa nacional) anunciadas por Zhou Enlai en 1974 bajo la idea-fuerza del Socialismo con Características Chinas, lanzada por Deng Xiaoping en $1982^{12}$.

El aspecto esencial para la comprensión de las reformas económicas chinas reside en el particular del proceso de desarrollo del país. China post-1978 puede ser percibida como uma variante de vía americana ${ }^{13}$, es decir, un proceso de industrialización apoyado en una amplia economía de mercado que se expande del campo a las ciudades cuyas

\footnotetext{
${ }^{12}$ Xiaoping (1992). No vamos a discutir en este artículo la conceptualización o incluso la validez teórica del "socialismo de mercado" como categoría de análisis. Se observa la categoría como una "formación socioeconómica distinta" o "Moderno Modo de Producción" (Gabrielle \& Schettino, 2012). Además, el socialismo de mercado deve ser observado como un modo de producción complejo que espeja, de un lado, supervivencia y fortalecimiento de instituciones de tipo socialista tradicional con el objeto de una estrategia nacional de carácter socializante operando y dirigiendo el cuerpo nacional y social en una formação social, también compleja, en que diferentes modos de producción no coetáneos conviven en contemporaneidad territorial y social bajo la hegemonía de la gran propiedade estatal socializada, espina dorsal de la economía y de la propia sociedad china. En este caso, la visión de totalidad histórica, muy cara a la ciencia de la Economía Política, puede sustituir cierto grado de primarismo y vícios neoclásicos que caracterizan la experiencia china de desarrollo como más una "transición en línea recta del socialismo al capitalismo" o una variante asiática, "sostenible" o no, de Estado desarrollista o un simple ejemplo de "capitalismo de Estado".

${ }^{13}$ Sobre la "vía americana de desarrollo" léase: Lenin (1915 [1964]) y Moore Jr. (1966).
} 
inducciones de tipo institucional potencian la transformación de productores pequeños y medianos en potenciales industriales, en una especie de acumulación sin expropiación (Arrighi, 2007, p.361-367) ${ }^{14}$, causa y consecuencia de la propia naturaleza gradualista de las reformas chinas. Este gradualismo tiene expresión en la combinación de los siguientes procesos liderizados pelo Estado: 1) de dominio del mercado por el Estado; 2) que a su vez liberaliza el comercio a su satisfación, abriendo posibilidades de profundización a formas superiores de división social del trabajo, y en concomitancia con el plan; 3) direcciona energías a la formación de un mercado de consumidor interno; 4) enceta la industrialización basada en el propio emprendimiento campesino: 5) induce la amplia competencia entre pequeñas, medianas y grandes empresas y estimula la educación como base atenuante de los efectos del propio mercado sobre el cuerpo social, y 6) planifica saltos, primero a la propia industria y en seguida al comercio exterior, no tradicional y sí como bien público, planificado y de Estado.

Este proceso, en China, tiene intimidad con el propio marchar de las reformas rurales, anabolizadas por dos elevaciones en los precios agrícolas y el consiguiente aumento de productividad y renta, además de la autorización -desde 1983- de búsqueda de mercado, por los campesinos, fuera de sus aldeas (Arrighi, 2007, p.366-367). He aquí el inicio de una nueva configuración de la división social do trabajo, bien como de esquemas regionales articulados tanto con la economía nacional, como con la internacional.

El principal ejemplo de esta dinámica está inserta en la transformación de la naturaleza del empleo en el país y su relación directa con el mayor poder de decisión económica a las provincias y a las empresas, la liberación de la mano de obra excedente de la agricultura y el fortalecimiento de industrias de carácter rural conocidas como Townshipsand Village Enterprises (TVE's). Una de las características fundamentales del proceso de desarrollo reciente chino está en el carácter rural de la gran manufactura expandida en la década de 1980. Las elevaciones de la renta y de la productividad del trabajo agrícola -y el consiguiente aumento de la demanda por bienes industriales- fue factor de deslplazamiento de mano de obra sobrante no a las grandes ciudades litoráneas y sí al propio alrededor, en el llamado poblado. Conformando, así, una más amplia división social/regional del trabajo fundada en la transferencia de actividades industriales

14 Esta noción de "acumulación sin expropiación" como forma de cacarcterizar la etapa inicial de las reformas económicas chinas puede servir a la demonstración para quien la propia expropiación ya había ocurrido en 1949. Sobre esto léase: Oliveira (2005). 
otrora ubicadas en el complejo comunal al ámbito del propio poblado, abriendo relieve a las tres principales características de la expansión de la economía de mercado, a saber: 1) especialización productiva; 2) diferenciación entre agricultura e industria y 3) superación de las barreras inhibidoras a la conexión de mercados regionales en un único e integrado mercado nacional.

La expansión de las TVE's da guarida a la toma de la división social del trabajo como una de las categorías nodales de análisis a la comprensión del desarrollo chino. En 1978, el número total de empleados en las TVE's era de 28,265 millones de trabajadores, triplicando en los diez primeros años de reformas económicas a 93,667 millones y llegando a 138,661 millones de empleados en 2004 (NBSC, 2005). Entre 1978 y 2004, la caída de la participación del empleo agrícola en China fue del $242 \%$, mientras el empleo rural no agrícola se elevou en el $471 \%$ en la industria, el $582 \%$ en la industria de la construcción y encima del $3.000 \%$ en el sector de servicios (Kang, 2006, p.291). Esta transferencia de actividades tuvo incidencia directa en la estructura de renta campesina: em 1978, el 7,92\% de la renta provenía de actividades no agrícolas, saltando al 30,61\% en 1996 con consecuente caída de renta vía actividades agrícolas cayendo de 90,08\% en 1978 al 69,39\% en $1996^{15}$. Cabe destacar, concomitante a esta expansión industrial en el campo, la producción de cereales ha tenido crecimiento continuo desde 2004, fruto de reformas institucionales que incluyó la abolición de todos los impuestos sobre la renta y la producción agrícolas, además de un claro proceso de alta mecanización y especialización productiva ${ }^{16}$. La producción de cereales en 2014 alcanzó el récord de 607,1 millones de toneladas (NBSC, 2015).

El reordenamiento de la división social del trabajo y el rumbo de la unificación del mercado nacional chino tuvo en las TVE's un elemento esencial. Sin enbargo, su radio de acción acompañó la propia estrategia de Estado, pasando a ser puente de la inserción internacional del país, sobre todo a partir de la década de 1990, siendo que al final de la década mencionada, las TVE's eran responsables por el $40 \%$ de toda la producción industrial (Masiero, 2006, p.432) y el 27\% de las exportaciones del país (Kang, 2006, p.137). En 1989, la exportación de textiles y calzados por las TVE's correspondió al $47,7 \%$ de las exportaciones de estos productos, cifra que cayó al 29,1\% en 2002 y al 23\% en 2007; mientras la participación en las exportaciones de bienes duraderos de consumo

\footnotetext{
${ }^{15}$ Ministry of Agriculture: Agriculture Development in China, 1995-1996.

${ }^{16}$ Sobre el proceso de especialización productiva y mecanización en la agricultura china, léase: Espíndola (2007).
} 
salió del 14,4\% en 1989, llegando al 29,1\% en 2002 y al 30,3\% en 2007 (NBSC, 2008). Ejemplos de expansión global de TVE's son innumerables, entre ellos se puede citar a: Haier con el 50\% del mercado de pequeños frigoríficos de los EE.UU.; a Galanz, con el $33 \%$ del mercado mundial de microondas; a Legend, con el 20\% del mercado mundial de placas para computadoras (ordenadores), y China International Marine Containers con un 40\% del mercado internacional de contenedores refrigerados (Masiero, 2006, p.441).

Los primeros pasos de las reformas económicas crearon condiciones materiales y políticas a pasos estratégicos acondicionadores de elecciones activas del país ante la creciente integración productiva y financiera global. Los bancos norteamericanos pasaron a acumular poder de gestión de la moneda de reserva internacional con musculatura creciente ante la tarea de capitanear intenso proceso de liberalización financiera, especialmente en los países de moneda no conversible (Belluzzo, 2012, p.130). Esta misma movilidad fue conferida al capital productivo de buscar mejores condiciones de reproduciones más allá de las fronteras de América del Norte en movimiento de causa y efecto de políticas monetaristas con impactos en el costo de la moneda y en la creciente valorización cambiaria. Es importante recordar que a principios de la década de 1980, las tasas de interés alcanzaron noveles sin precedentes en los EE;UU., en la casa de los dos dígitos (Galbraith, 1987, p.247). Además, la ofensiva norteamericana sobre las condicionantes macroeconómicas de la economía japonesa (Acuerdos de Plaza de 1985) aceleraron el proceso de reconfiguración de la división internacional del trabajo bajo mando de las grandes finanzas, promoviendo reajustes geográficos que, en última instancia, beneficiaron la mediación entre los objetivos estratégicos chinos y la propia política de internalización de tecnologías y métodos avanzados de gestión de la producción.

Las reformas económicas en China y su Economía Política son, también, la historia de la creación de condiciones objetivas a la solución de pendencias históricas, especialmente Kong-Kong, Macao y Taiwán. No es de sorprenderse que el objetivo primordial del financiamiento de proyectos y formación de grandes empresas conjuntas (joint ventures) estaba en la movilización de inmensos recursos financieros de chinos de ultramar. Un círculo internacional chino formado se convirtió en una externalidad virtuosa anterior a la afluencia de IED's de los EE.UU., la Unión Europea y Japón, como el primer paso hacia la propia integración productiva asiática centrada en China, en un movimiento de desplazamiento del antiguo centro japonês. Entre 1990 y 2008, el $45 \%$ de los IED's realizados en China tenía su origen en el complejo Hong-Kong / Macao; en 
segundo lugar venían los EE.UU., empatados con Taiwán, con un 9\%; seguidos por Singapur, con el 6\%, y Alemania y Francia correspondían al $2 \%$ y el 1\%, respectivamente (Jabbour, 2012, p.238). Si se toma, en conjunto, el complejo Hong-Kong / Macao, Taiwán y Singapur, se concluye que más del 60\% de los IED's en China son ejecutados por chinos. Se Trata de aspectos fundamentales de análisis antecesoras tanto del boom de las exportaciones como de la alta relación inversión x PIB ocurridos a partir de la segunda mitad de la década del 1990. Y, sobre todo, tras la visita histórica de Deng Xiaoping, en 1992, a las provincias prósperas del sur, que este movimiento de captación financiera y productiva de la diáspora se efectúa, de hecho. La inexorabilidad de las reformas económicas, amenazadas tras el fin de la Unión Soviética, se convierte en un hecho político y estratégico con la proclamación del socialismo de mercado como objetivo mater del régimen en 1993.

\section{LA MACRODINÁMICA Y LA FORMACIÓN DE UNA POTENCIA FINANCIERA}

Las primeras etapas de las reformas econômicas se caracterizaron por un aumento de la renta campesina acompañada por un aumento de la demanda por bienes de consumo. La elevada tasa de crecimiento ocurrida entre los años 1978 y 1984 fue acompañada por cambios estructurales en los patrones de consumo (Medeiros, 1999, p.96), con una ampliación de horizonte consumista que pasó a ser pautado por la adquisición de televisores, heladeras, relojes y lavadoras, con la consiguiente explosión de la producción interna de estos productos, especialmente en las TVE's ${ }^{17}$.

Así, la participación del sector primario en la composición del PIB pasó del 28,2\% en 1978 al 32,1\% en 1984, mientras el sector secundario cayó del 47,9\% al 43,1\%, habiendo un pequeño crecimiento del sector terciario del 23,9\% para el 24,8\%. El empuje del sector primario al resto de la economía perduró, aún, hasta el 1990, cuya contribución al crecimiento general del PIB fue del 41,7\%, mientras que la industria agregada al sector de la construcción tuvo participación del $41 \%$ y el sector de servicios, el 17,3\%. Esta tendencia sólo se supera después del 1995, cuando la contribución de la agricultura a la elevación del PIB cayó de forma acentuada al 9,1\%, la industria y el sector de la construcción llegó al 64,3\% y los servicios al 26,6\%. En 2007, la claridad dela primicia de las inversiones sobre la demanda total queda evidente con la participación de la

\footnotetext{
${ }^{17}$ Esta discusión sobre el cambio de los patrones de consumo y consiguiente elevación de la producción interna de bienes destinados al consumo de masa es muy desarrollada en: Sing (1993).
} 
agricultura cayendo, aún más, al 3,6\%, la industria (más construcción) alcanzando el $54,1 \%$ y los servicios, el 48,2\% (NBSC, 2008).

La transición entre un patrón de crecimiento basado en el consumo (desde principios de la década de 1980) a otro combinando aumento rápido del producto por la vía de las exportaciones y de la inversión se consolida en la mitad de la década de 1990. La formación bruta de capital fijo, en la década de 1980, tuvo un promedio del 35,7\%, alcanzando el actual nivel -por encima del 45\%- solamente en 1995, y pico del 48,7\% en $2011^{18}$. Las exportaciones salieron del 10\% del PIB en 1978, llegando al 17\% en 1984 y al 45\% en 1995. A nivel internacional, el peso de las exportaciones chinas en relación a las mundiales, que en 1978 fuera del 0,75\%, en 1995 alcanzó el 3\% (Medeiros, 1999,p.96), 6\% en 2007 y 11,3\% en 2013 (WTO, 2014). El comportamiento de la inflación en los primeros 15 años de las reformas acompañó la tendencia de una economía de consumo en expansión mediada por bajo control oligopólico, liberalización de precios y utilización de capacidades productivas instaladas (TVE's). La inflación sufrió fuertes oscilaciones entre 1978 y 1995, con picos en 1981, 1985, 1988 y 1994, cuando llegó al $24 \%$ al año (Nonnemberg, 2010, p.207). La alta tasa de inversiones fue factor de gran importancia a un equilibrio dinámico inflacionario medio por debajo del 4\% entre 1996 y 2014, incluso a la actual posibilidad de tendencia desinflacionaria.

Volvamos al comportamiento de la tasa de inversiones. Su ascensión coincide con dos movimientos, el primero es función del proprio cambio de patrón de acumulación consagrado en la década de 1990 (export-led). El segundo, derivado de la continentalidad y la consiguiente necesidad de integración de pequeños mercados al todo complejo regional, que a su vez avanza en el rumbo de un gran mercado nacional aún en vías de consolidación; elemento casi ausente en los congéneres desarrollistas del Este asiático cuyos picos en la relación entre inversión x PIB no pasaron el 39\% (Corea del Sur en 1991). En este sentido, el desarrollismo y su adaptación a una economía continental, y viceversa, deben ser observados a lo largo del tiempo y percibida como parte de la estrategia cuyo movimiento expresa la propia estrategia, como mostraremos a continuación.

\footnotetext{
${ }^{18}$ En 2014 esta tasa fue del 46,1\%.
} 


\section{Estrategia y dinámica regional}

La liberación de fuerzas de mercado engendraba, en su contenido, nuevas configuraciones en materia de planificación económica que sólo ganan forma y contenido a principios de la década del 2000 con un intenso proceso de fusiones y adquisiciones en el sector estatal de la economía y la formación de un ambiente institucional y, sobre todo, financiero capaz de cimentar una estrategia de inserción internacional y saltos internos de un desequilibrio a otro.

Preparar el territorio chino a aprovechar la tendencia de las grandes corporaciones de obtener ganancias crecientes en escala y reducción de costos fue la primera gran prueba de preparación del país a la admisión soberana en las cadenas globales de valor. Parte de un todo complejo que relaciona la internalización de un modelo export-led con la necesaria construcción de una soberanía financiera (reservas cambiales) central a la consecución de una política de largo alcance basada en mecanismos de socialización de la inversión ${ }^{19}$, que se revela a lo largo de los últimos 30 anos el pilar central de coordinación entre capital estatal, concentrado y centralizado, e intensa capilaridad e importancia del sector privado de la economía china. Se trata de una virtuosa que explica el dinamismo económico chino en la maximización de los sectores estatal y privado y de la relación de unidad de contrarios entre a planificación de nivel superior y el creciente papel del mercado en las decisiones a nivel de la firma.

La institución de las Zonas Económicas Especiales (ZEE's) atendía a una estrategia de inserción económica mundial del país, de la misma forma que iban al encuentro de la creación de una zona de convergencia económica entre China-HongKong y China-Taiwán, paso primero a la reunificación del país bajo el aliciente de la política de "un país, dos sistemas". Las cinco primeras experiencias instituidas entre 1982 y 1984 fueron escojidas estratégicamente: Shantou, Shenzen y Zhuhai en la provincia fronteriza con Hong-Kong y Xiamen, en la provincia de Fujian (al frente de Taiwan) y toda la isla de Hainan, más al sur y cerca de los chinos étnicos establecidos principalmente en Singapur e Indonesia. Shangai fue restablecida como la cabeza del dragón, nuevo centro industrial y financiero del país y sede de la Zona Económica Especial de Pudong,

\footnotetext{
${ }^{19}$ Sobre la noción de "socialización de la inversión”, la tomamos conforme Keynes (1964, p.378): “( ...) the state will have to exercise a guiding influence on the propensity to consume partly through its scheme of taxation, partly by fixing the rate of interest, and partly, perhaps, in other ways (...). I conceive, therefore, that a somewhat comprehensive socialization of investment will prove the only means of securing an approximation to full employment; though this need not exclude all manner of compromises and of devices by which public authority will co-operate with private initiative".
} 
ubicada al margen oriental del río Pudong, formada para albergar las oficinas de P\&D de las mayores corporaciones industriales chinas y del mundo (Oliveira, 2003, p.9).

La concepción de esta dinámica territorial gradual está en los escalones de desarrollo frente a frente con la ley de desarrollo desigual, en que se identifica la formación de un centro dinámico y posterior incorporación de la perifería a este centro común a países de nivel medio de desarrollo y alto grado de catch up-. Tal es la política regional oficial del Estado chino desde el VII Plan Quinquenal (1986-1990) engendrando - por consiguiente- inmensa concentración territorial de renta como sustituyente a la duplicidad de mundos entre el urbano t rural mediado pelo sistema de control de migración interna (Hukou) de la era maoísta.

Las ZEE's tuvieron su experiencia prolongada a todo el litoral (1987), además de 2000 ciudades de frontera en 1992, aparte de la autorización a 30 provincias de elaboración de sus propias políticas de apertura (Demurger et al., 2002, p.157). El primer gran movimiento de reversión de políticas positivas al litoral fue la asunción de Chongqing, ubicada en el medio oeste y ramificación / cruce del río Yangtsé a la condición de municipalidad, como Pekín, Shangai y Tianjin. Este movimento es similar al verificado en la segunda mitad del século XIX en los EE.UU. y su estrategia de expansión al oeste centrada en la ciudad de Chicago, medio oeste del país y punto de cruce ferroviario y rodoviario. Entre enero de 2001 y diciembre de 2010, US\$ 1,8 mil millones mensuales fueron asignados en la municipalidad de Chongqing bajo la clara estrategia de transformarla en la "Chicago China", incluyendo la formación y el desarrollo de instituiciones similares a las creadas en los EE.UU. en el último tercio del siglo XIX, a saber: Tennessee Valley Authority, Civilian Conservation Corps y la Interstate Highway System (Jabbour, 2006, p.78).

Bajo la ideia fuerza del "Programa de Desarrollo del Gran Oeste" (1999), se intenta consolidar un gran mercado nacional y la viabilidad de conectar la economía continental china a sus vecinos, bajo el propósito de la transferencia de renta del litoral en el intercambio de materias primas abundantes en el centro y oeste del país. Cerca de 100 mil millones de dólares fueron asignados, sólo entre 1999 y 2005, en grandes proyectos infraestructurales de largo alcance, siendo las dos principales el gasoducto Oeste-Este (Xinjiang-Shanghai) y el ferrocarril Qinghai-Tíbet (Jabbour, 2006, p.60). Las inversiones en activos fijos con relación al PIB en la región Este del país cayeron del 54\% al 41\%, entre 1998 y 2013. Mientras que en las regiones Noreste, Oeste y Central salieron del nivel del $8 \%, 18 \%$ y $17 \%$ en 1998 al $10 \%, 25 \%$ y $24 \%$, respectivamente (NBSC, 
2015). La renta per cápita del Oeste en proporción a la renta per cápita nacional salió del nivel del 90\% en 1990, cayendo al 81,3\% en 1998 e iniciando tendencia de aproximación con la nacional, alcanzando 84,2\% en 2013 (Morais, 2015, p.247). Del paquete de inversiones, anunciado en noviembre de 2008, de US \$ 600 mil millones, el $73 \%$ fue asignado en las regiones interiores del país.

De la implementación de las primeras ZEE's costeras al ambicioso "Programa de Desarrollo del Gran Oeste" en 1999, seguido por programas similares de inversiones con vistas a la quiebra de bloqueos de mercado lanzados hacia el Noreste (2002) y Centro (2004) del país, la configuración de una economía continental bajo las huestes de la formación de un gran mercado nacional unificado guarda correspondencia con el aumento de la influencia china en el mundo y, sobre todo, hacia sus vecinos al Oeste. La presencia continental china, además de sus fronteras, es clara en el ambicioso proyecto lanzado por el actual presidente chino, Xi Jinping, de reconfiguración de una Nueva Ruta de la Seda (Ren, 2015), con inversiones previstas para 2020 de 2,5 billones de dólares (Tiezzi, 2015): cinturón económico conectando el mercado chino desde la costa hasta el conjunto de Asia Central, en clara respuesta a las objeciones norteamericanas en Oriente Medio y antiguas repúblicas de la extinta Unión Soviética.

Ejes regionales de desarrollo fueron estimulados, institucionalizados y sobre todo planificados desde 1982. El diseño de peldaños de desarrollo guarda objetividad en la tendencia de dispersión regional de inversiones e industrialización, tras la segunda mitad de la década de 1990. Se trata, también, de un proceso de transformación del desarrollo económico en desarrollo territorial, además forma de ejecución de una gran estrategia nacional y de constante legitimación del propio poder político instalado en octubre de 1949. En este sentido, la creciente demanda china por hidrocarburos coincide con el descubrimiento de grandes reservas de petróleo y gas natural en el Xinjiang ${ }^{20}$, haciendo estratégica esta región autónoma tanto como suministradora de energía como cabeza de puente entre China y sus cercanías petroleras de Asia Central. Tensiones étnicas en esta región huigur recrudescieron desde la década de 1990 e inicio del presente siglo, momentum histórico de simultaneidad con inversiones de -entre 1990 y 2001- 15 mil

\footnotetext{
${ }^{20}$ Estas reservas se estiman en cerca de 3 mil millones de toneladas de petróleo y 1,3 billón de metros cúbicos de gás natural, comprobados, pero estimados entre 20 y 40 mil millones de toneladas de petróleo y 12,4 billones de metros cúbicos de gas natural (Bandeira, 2013, p.125).
} 
millones en infraestructura, además del movimiento de transformar Xinjiang en el mayor complejo petroquímico de Asia hasta 2020 (Bandeira, 2013, p.125).

\section{La dinámica y el papel del Estado}

A nuestro juicio, no quedan dudas sobre la centralidad del papel del Estado en el esfuerzo concentrado de modernización y admisión plena de China en el rol de las grandes potencias económicas mundiales. Sin Embargo, este papel no puede ser analizado de forma estilizada, basadas en nociones estáticas del proceso. En este particular reiteramos la ciclicidad del papel del Estado en China, así como -en dinámica- la percepción de diferentes roles otorgados a éste a lo largo del proceso, encaminando la elaboración / ejecución de nuevas y superiores formas de planificación económica vis à vis como el núcleo gestor de mecanismos de socialización de la inversión.

El argumento ortodoxo sugiere una dinámica liberalizadora, como sigue: desmonte de los complejos comunales rurales, concesión de tierras a las familias campesinas y consecuente recomposición de la pequeña producción mercantil (laissezfaire campesino). Liberalización que se extiende a la industria y servicios hasta alcanzar el propio sistema de formación de precios. Apertura del país a la IED’s y admisión plena a la Organización Mundial do Comercio (OMC) en 2001. Privatizaciones masivas de activos estatales ocorridas entre 1994 y 2000 y la propiedad privada reconocida, legitimada y sostenida por leyes y reglamentos (Naughton, 2006, p.2). Concomitante, el número de quiebras en el sector estatal alcanzó 1.232 empresas en 1995, 4.198 en 1998 y 5.429 en el 2001 (Imai, 2006, p.5).

Entre 1998 y 2007 el total de empresas estatales en China cayó un 39,2\% del total de las empresas al 6,1\%, mientras que el sector privado salió, en el mismo período del $6,5 \%$ del total al $52,6 \%{ }^{21}$. El carácter intensivo en trabajo de muchas empresas estatales llevó a una no completa proporcionalidad de caída en relación a la verificada en el número de empresas de tipo industrial / urbano. En el período indicado la caída del número de trabajadores en el sector fue del $168 \%$, pasando, así, a responder por el $29,5 \%$ del total de los puestos de trabajo mientras el sector privado sale del 2,6\% en 1998 al 28,6\% en 2007 (Jabbour, 2012, p.238). No cabe duda de que bajo un objetivo puramente hipotéticodeductivo, estos datos dan pleno contorno, y argumento, ante el gran número de quiebras de empresas estatales y aumento de importância del sector privado, a la ortodoxia.

\footnotetext{
${ }^{21}$ Tratamos aqui solamete de las empresas con capital total estatal o privado. Otra característica china es la multiplicidad de formas de propiedad, especialmente mixtas.
} 
El proceso de aumento de importancia absoluta del sector privado guarda gran consecuencia e, incluso, imperativo, a la conformación de una larga economía de mercado. Los crecientes excedentes en la agricultura sirvieron de nicho mercantil rápidamente ocupado por las TVE's, cuyo dinamismo produjo un fuerte impacto negativo sobre, principalmente, las pequeñas y medianas empresas estatales ocupadas en la producción de bienes de consumo. Estas estatales fueron desarrolladas lejos de métodos modernos de gestión y subordinadas a un esquema central y débil de planificación de producción de tipo soviético, caracterizadas por métodos rústicos e ineficientes de sustitución del cálculo económico e inexistencia de relações upstream y downstream entre la propia empresa, ancilares y consumidores. Por otro lado, la disminución cuantitativa del sector estatal tuvo compensación social nada modesta de creación de millones de empleos en las zonas rurales y urbanas, además de una dinámica clase de capitalistas domésticos de inmensa funcionalidad a la estrategia nacional e internacional china.

Y el actor clave de esta estrategia de desarrollo, en este caso, es el propio Estado. La combinación entre concentração de mercados e descentralización del plan (Medeiros, 1999, p.109) tiene numerosas facetas sintetizadas en una combinación única entre mercado e planificación que caracteriza la vía china de desarrollo hasta los días actuales, conformando nuevas y profundas formas de división social del trabajo. El mayor poder de decisión atribuido a las provincias y la libertad casi completa de acción concedida a las TVE's fueron causa y consecuencia de una política fiscal, inaugurada, en 1980, marcada justamente por renuncias fiscales en favor de la retención de ingresos a nivel provincial acarreando en crecientes déficits fiscales hasta $1994^{22}$. La transición de una economía centralmente planificada a otra más flexible, con tendencia estratégica de fusión entre grandes empresas y grandes bancos, demandó reformas iniciando la optimización de mecanismos de control macroeconómicos (Ki; Yuk-Shing, 1994, p.769). Estas reformas en China antecedieron a las transformaciones necesarias al sector estatal industrial creando condiciones institucionales, por ejemplo, al enfrentamiento de la actual

\footnotetext{
${ }^{22}$ Es temerario no relacionar esta mayor autonomía local con la lucha entre reformistas y conservadores en el seno del PCCh encerrada con la completa victoria del ala reformista liderado por Deng Xiaoping y consagrada en el $14^{\circ}$ Congreso Nacional del PCCh (1992). La mayor autonomía a los líderes locales para llevar adelante reformas y experimentos económicos fuera de amarras ideológicas, además del apoyo del Ejército de Liberación Popular (ELP) a cambio de mayor autonomía empresarial al ELP se constituyeron en los dos pilares políticos que hizo posible el mantenimiento y profundización de las reformas económicas en el inicio de la década de 1990. Sobre este interesante proceso político, léase: Martí (2002). Sobre los déficits fiscales acumulados entre 1979 y 1994, léase: Ki y Yuk-Shing (1994).
} 
crisis financiera internacional. En este sentido, la elevación del papel del mercado y una mayor participación del sector privado, incluso sustituyendo el sector estatal en determinadas áreas, pasó a tener fuerte contrapartida en el anuncio, en 1994, de reformas en el sistema tributario que apuntaron en la dirección de un fuerte proceso de recentralización y concentración. Recentralización fiscal y mayor poder estatal sobre los mecanismos macroeconómicos esenciales se tornaron el paso primario hacia la institución de mecanismos de coordinación y socialización de la inversión. China daba un paso decisivo a la internalización de una moderna economía monetaria.

La exposición a la dura competencia ante las TVE's y a un pujante sector privado emergente expuso la demanda urgente de reformas profundas en las empresas estatales de forma a legitimar su papel de espina dorsal del todo complejo sistema empresarial ya diseñado en mediados de la década de 1990 en el seno del establecimiento, como objetivo estratégico del régimen, de una economía de mercado socialista. Al menos dos grandes movimientos en este sentido deben ser destacados: 1) el movimiento de centralización y concentración del gran capital estatal, y 2) profundización del proceso de separación entre gestión y propiedad abriendo camino a diversas modalidades de propiedad pública.

El sector estatal industrial da economía acumuló pérdidas anuales entre 1991 y 1999 del orden del 0,6\% del PIB, marco completamente inverso en 2007 con ganancias que corresponden al 4,2\% del PIB (Gabriele, 2009, p.7). La comparación de la productividad del trabajo / ingreso por trabajador entre las empresas estatales y privadas, es sugestiva. En el año 2007, esta relación ${ }^{23}$ en las empresas estatales fuera de 58,3 / 84,7; en las TVE's, 41,2 / 24,8; en las empresas privadas 48,1 / 32,5 y en los conglomerados estatales 68,7 / 90,8; en las empresas de capital mixto con participación estatal mayoritaria, 88,0 / 99,2 y en las empresas de capital mixto bajo control privado, 41,6 / 90,8 (Jabbour, 2012, p.190). Aunque, a menudo la inmensa maioría de las empresas chinas estén bajo control del sector privado, el poder económico continúa firmemente concentrado en las manos del Estado. Ejemplo de ello está en la íntima relación entre grandes empresas privadas y su relación cercana con el Estado por medio de participación accionaria del propio Estado, crédito y completo aporte estatal a sus estrategias de inserción internacional.

La transición, ya aludida, de una economia de mando a la otra con mayor papel concedido al mercado y en que el Estado pasa a ser el núcleo gestor de políticas de

\footnotetext{
${ }^{23} 10.000$ yuanes por unidad.
} 
socialización del proceso de inversión gana cuerpo con la formación, en 2002, de SASAC (State-Owned Assets Supervision and Administration Comission), creada en el sentido de representar los intereses del Estado, y de sus acciones, en los 149 principales compañías del país, concentradas en los sectores con alto grado de oligopolio / monopolio ${ }^{24}$. Esto significa, na ponta do processo, na transformação destas corporações no núcleo-duro executor das políticas oficiais de Estado, conforme o grande papel conferido a estas companhias nos imensos programas de investimento executados no bojo à resistência aos efeitos da crise financeira internacional ${ }^{25}$.

\section{La gran finanza nacional}

La creciente influencia política y económica de China sobre el mundo guarda gran evidencia en la relación entre grandes reservas cambiarias y la actual posición de mayor acreedor neto del mundo al valor de 1,97 billón de dólares, lo equivalente al 20,8\% de su PIB (Cintra; Silva Filho, 2015, p.426). El país transformó su estrategia internacional en una cuestión que abarcaba la fusión entre la gran empresa con la gran finanza, explorando el grado de madurez alcanzado por su sistema financiero, alzando el país al nivel de potencia financiera.

Colocarse en el mundo como una potencia financiera ha sido consecuencia lógica de un proceso mercantilista moderno de transformación tanto en potencia comercial como en una gran fábrica del mundo. La remodelación de funciones entre Estado e iniciativa privada forjó instituciones del nivel de la propia SASAC, cuya contraparte financiera fue la constitución de una moderna economía monetaria en sustitución de rústicas formas bancarias, inherentes al modelo soviético, mediadoras de ahorro forzado, en un proceso de continua evolución institucional que moldeó una gran finanza funcional a objetivos estratégicos de Estado. La combinación entre coordinación de la inversión (SASAC) con un sistema estatal de intermediación financiera conforma el nivel superior de atuación estatal, tanto como prestamista de última instancia como el propio inversionista de primeira instancia (Burlamaqui, 2015, p.47).

Se trata, por lo tanto, de elemento con grados variados de funcionalidad, que se entrecruzan, a saber: 1) impacto decisivo sobre las condiciones cada vez más complejas

\footnotetext{
${ }^{24}$ Un rápido y amplio proceso de centralización y concentración en el marco del gran capital estatal ocurrió, principalmente, tras la reforma fiscal promulgada en 1994. La formación de 149 conglomerados empresariales estatales fue el punto culminante de este proceso.

${ }^{25}$ Sobre los principios y acción de SASAC, léase: Naughton (2006).
} 
de financiamiento de la economía, y, consecuentemente, sobre el nivel de gastos de los agentes y afectando a las variables reales de la economía, como producto y empleo (Paula, 2014, p.2), y 2) elemento con papel creciente ante el grado de intervención de Estado en una economía continental, sujeta a emergencias constantes de bloqueos de mercado, que a su vez tiene en la transferencia intersectorial de recursos el principal medio de enfrentamiento a los constantes desequilibrios de variada monta sean sociales, regionales y entre los diferentes sectores de la propia economía. Otro punto de desequilibrio reside en las contradicciones relacionadas con la propia evolución del sistema financiero, entre ellas la propagación de una finanza paralela, no regulada, fuera de los balances de bancos comerciales y con cierta capacidad de profundizar desequilibrios en una economía aún fuertemente pautada por la inversión con la urgente necesidad de cambio de dinámica interna de acumulación ${ }^{26}$.

La evolución institucional acompañó e, incluso, se adelantó a las exigencias del proceso de reformas económicas, incluyendo la superación del alto grado de represión financiera. Entre 1978 y 1984 el Banco Popular de China elevó su papel a la propia regulación financiera, mientras cuatro bancos sectoriais fueron formados (Big Four) atendiendo a las exigencias anexas a la agricultura, construcciones urbanas, infraestructuras y financiamiento de exportaciones e importaciones ${ }^{27}$. El avance de la urbanización -incluyendo el financiamiento de grandes eventos como los Juegos Olímpicos de 2008, las políticas de desarrollo del oeste y la reacción coordinada entre el gobierno central y las provincias a la crisis de 2008- demandó la formación, ex ante, de grandes bancos provinciales y municipales de desarrollo forjadas concomitante con el proceso de fusiones y adquisiciones de pequeñas y mediadas empresas en la década de 1990 y el surgimiento, en el ámago, de 149 conglomerados estatales.

Todavía sobre la evolución institucional acelerada en la década de 1990, es menester notarla como parte de la propia antecipación de hechos que iría ocurrir en el seno de los acuerdos comerciales China-EE.UU. y la admisión del país a la OMC, en 2001. Pruebas de esta antecipación de hechos están en las sucesivas rondas de

\footnotetext{
${ }^{26}$ Nos referimos al llamado "sistema financiero sombra" ("Shadow Banking").

${ }^{27}$ Como se ha descrito, el Banco Popular de China es el responsable por la regulación del sistema financiero y rige, además, comisiones como la de Regulación Bancaria de China (CBRC), de Regulación de los Valores Mobiliarios de China (CSRC) y la de Regulación de Seguros de China (CIRC). Sobre la naturaleza institucional y política del Banco Popular de China, según Burlamaqui (2015, p.50): “(...) es una administración con status ministerial, que opera bajo el liderazgo del Consejo de Estado. (...) no hay independencia del Banco Central, sino articulación institucional con otros órganos políticos, bajo una agencia piloto, el Politburó, que a su vez está subordinado al PCCh”.
} 
capitalización sobre los bancos comerciales estatales mediadas por la emisión de títulos y compra, por compañías recién creadas, de créditos podridos con vistas a su gestión y recuperación ${ }^{28}$. Ambos movimientos que anunciabam el lento proceso de apertura del sistema financiero chino a la competencia y a la propia apertura de capital. Además, la crisis financiera asiática llamó la atención de las autoridades del país a los propios límites de la gran finanza interna, prontamente enfrentados.

En 2015, el sistema financiero pasó por una gran prueba, con caídas acentuadas verificadas en las bolsas domésticas, acelerando las especulaciones acerca de una crisis financiera que golpeó las puertas del país con efectos, hasta entonces inimaginables al resto do mundo. ¿Habría sido un gran canto de réquiem a un "modelo insostenible” y esgrimido en la fragilidad de una finanza interna susceptible a injunciones políticas, no "técnicas"? Sostenemos que es de grande superficialidad este tipo de narrativa, preferiendo situar la cuestión en el campo, como puesto en el inicio del artículo, del desgarramiento de una dinámica de acumulación pautada por bajas tasas de interés y mayor papel concedido a las provincias a la ejecución de amplios programas de infraestructuras en medio a la crisis internacional, exponiendo las propias fragilidades de la dinámica de acumulación y de su gran brazo financiero, también influenciado por agentes privados - causa y consecuencia del proceso de liberalización recientemente observado.

Es sugestivo situar el problema en el propio campo del desequilibrio estructural y en la necesaria conjunción de interpretación de fenómenos complejos. Por ejemplo, la visión que percibe el desequilibrio como premisa de la principal variable / categoría de validación teórica en el campo de las ciencias económicas, a saber, la planificación. Bajo este prima, ¿nuevos cambios institucionales -como los que siempre precedieron cambios recientes sobre la base económica del país- ya no estarían en curso, como se puede percibir en la propia rapidez con que entró y salió de los noticieros la entrada del país a una "crisis financiera"?

\footnotetext{
${ }^{28}$ Según Cintra (2015, p.404), "De hecho, las sucesivas rondas de capitalización operadas por el Estado prepararon los big four para la apertura de capital -lo que acurrió desde mediados de los años 2000 (...). En 2005, el CCB abrió su capital y captó US \$ 9,23 mil millones. En 2006, el BOC captó US \$ 11,2 mil millones, y en octubre de 2006, el ICBC realizó su oferta pública inicial de acciones, por el valor de US \$ 19,1 mil millones (...).”. Mayores detalles sobre este interesante y planificado proceso puede ser encontrado en: Allen; Qian; Zhang e Zhao (2012) e Brillant (2011).
} 


\section{CONSIDERACIONES FINALES}

A la luz de lo expuesto, de la historia reciente del proceso de desarrollo chino, ¿qué disponer para sugerir el propio futuro inmediato del país y sus desafíos?

Este artículo buscó exponer que la historia de las reformas económicas chinas tiene como característica fundamental la capacidad del Estado, y de su fuerza política gobernante (PCCh), de operar las transformaciones políticoinstitucionales necesarias a la consecución de una estrategia nacional de largo plazo. La principal transformación fue transmutación de una base económica moldeada por instituciones típicas del modelo soviético a la formación de una economía monetaria moderna capaz de proveer al Estado y sus agentes una amplia capacidad de coordinación y socialización de la inversión y, por consiguiente, margen de maniobra política y financiera al lanzamiento de nuevas y superiores formas de planificación económica; como las verificadas en las políticas de desarrollo y expansión regionales y en las grandes medidas de coordinación sobre los mecanismos de control de la gran finanza y sus fragilidades expuestas recientemente.

El país vive actualmente una transición de dinámica de desarrollo que trae consigo no solo las vicisitudes de un proceso de esta magnitud. Absorbe también una pléyade de contradicciones y desequilibrios en forma de crisis ambiental, desigualdades sociales, territoriales y de renta todavía explosivas, índices de consumo muy por debajo del nivel de los países desarrollados. Así como los desafíos externos inherentes a la lenta internacionalización de su moneda y de una política externa activa y basada en inmensas inversiones en infraestructuras por todo el mundo, especialmente en su entorno asiático (incluyendo la Nueva Ruta de la Seda), África e América Latina.

Hay impactos políticos a largo plazo a ser considerados en el análisis, entre tales la propia relación entre este creciente y pujante sector privado y el Estado. ¿Hasta qué punto el Estado conseguirá equilibrarse entre los intereses generales de la sociedad y los intereses particularistas ihnerentes al sector privado? ¿La intensa capilaridad e importancia de este sector y su expansión no sería capaz de -a largo plazo- limitar la propia capacidad del Estado de coordinar grandes decisiones de inversión?

Se trata de una gama de desequilibrios interconectados. Lo que está en cuesión, de hecho, no es sólo el destino de la economía. Y sí, de la propia Revolución China.

\section{REFERENCIAS BIBLIOGRÁFICAS}

ALLEN, F.; QIAN, J.; ZHANG, C.; ZHAO, M. (2012): “China's financial system: opportunities and challenges”. NBER WorkingPaper 17828. 
ARRIGHI, G. (2007): Adam Smith in Beijing - Linkagesofthe 21th Century. New York: Verso.

BANDEIRA, L. A. M. (2013): A Segunda Guerra Fria - Geopolítica e Dimensão Estratégica dos Estados Unidos. Rio de Janeiro: Civilização Brasileira.

BELLUZZO, L. G. (2012): O capital e suas metamorfoses. São Paulo: UNESP Editora. BRESSER PEREIRA, L. C. (2006): Estratégia Nacional e Desenvolvimento. Revista de Economia Política, 26, 2 (102) : pp. 28-42.

(1989): "O caráter cíclico da intervenção estatal”. Revista de

Economia Política, (9) 3: pp. 28-42.

BRILLANT, G. (2011) "Systeme bancaire chinois: entre controle de l'État et adaptation aux normes internationales". Revue d'EconomicFinancière, 102: pp. 43-56.

BURLAMAQUI, L.; SOBREIRA, R.; VIANNA, M. (2015): The Present and Future of Development Financial Instituitions. Rio de Janeiro: Minds.

CINTRA, M.; SILVA FILHO, E. (2015): "O Sistema Financeiro Chinês: A Grande Muralha". In, CINTRA, M.; SILVA FILHO, E.; PINTO, E. (2015): China em Transformação: dimensões econômicas e geopolíticas do desenvolvimento. Rio de Janeiro: IPEA.

DÉMURGER, S.; SACHS, J; WOO, W. T.; BAO, S.; CHANG, G.; MELINGER, A. (2002): "Geography, Economic Policy and Regional Development in China". Asian Economic Papers, 1 (1): 146-197.

DOMAR, E. (1972): “A Soviet Modelof Growth”. In, NOVE, A.; NUTI, D. M.: Socialist Economics. Penguim.

ESPÍNDOLA, C. (2007): "Notas sobre o agronegócio de carne na China". Revista de Geografia Econômica, Edição Piloto: 210-219.

GABRIELLE, A. \& SCHETTINO, F (2012). "Market Socialism as a Distinct Socioeconomic Formation Internal to the Modern Mode of Production". New Proposals: Journal of Marxism and Interdisciplinary Inquiry. v. 5 (2): 20-50.

GALBRAITH, J. K. (1987): Economics in Perspective: A Critical History. New York: Hardcover.

JABBOUR, E. (2012): China Hoje: Projeto Nacional, Desenvolvimento e Socialismo de Mercado. São Paulo: Anita Garibaldi/EDUEPB.

(2006): China: infra-estruturas e crescimento econômico. São Paulo:

Anita Garibaldi.KANG, H. (2006). China's Township and Village Enterprises. Beijing: Foreign Language Press.

KEYNES, J.M. (1936 [2003]). The General Theory of Employment, Interest and Money. Basingstoke: Palgrave MacMillan.

KI, S.; YUK-SHING, C. (1994): “China's Tax Reforms of 1994: Breakthrough or Compromise?", Asian Survey, (34) 9: 769-788.

KISSINGER, H. (2011): On China. New York: New York Penguin Press.

IMAI, K. (2006): "Explaining the Persistence of State Ownership in China". Institute of Developing Economies, Discussion Paper 64 (5):1-23

LENIN, V. (1921 [1964]): "The Tax in Kind (The Significance Of The New Policy And Its Conditions)". In, LENIN, V. (1964): Collected Works. Moscow. Progress Publishers. v 32: 326-365.

(1915 [1964]): "New Data on the Laws Governing the Development of Capitalism in Agriculture - Part One: Capitaism and Agriculture in United States of America". In, LENIN, V. (1964): Collected Works. Moscow. Progress Publishers. v 322: 13-122.

LOSURDO, D. (2004): Fuga da História? A Revolução Russa e a Revolução Chinesa vistas de hoje. Rio de Janeiro: Revan. 
MASIERO, G. (2006): "Origens e desenvolvimento das Township and Village Enterprises (TVE's) chinesas”. Revista de Economia Política, 263 (103): 425-444.

MEDEIROS, C. A; CINTRA, M. R. V. P. (2015): "Impacto da ascensão chinesa sobre os países latino-americanos". Revista de Economia Política, 35, 1 (138): pp. 28-42.

(1999): "Economia e Política do Desenvolvimento Recente da China".

Revista de Economia Política, 19, 3 (75):92-112.

MARTÍ, M. E. (2002): China and the Legacy of Deng Xiaoping - From Communist Revolution to Capitalist Evolution. Lincoln: Potomac.

MOORE JR, B. (1966): Social Origins of Dictatorship and Democracy: Lord and Peasant in theMaking of the Modern World. Boston: Beacon.

MORAIS, I. N. (2015): "Desigualdades e Políticas Públicas na China; investimentos, salários e riqueza na era a sociedade harmoniosa". In, CINTRA, M. A. M.; FILHO, E. B.; PINTO, E. C. (2015): China em Transformação: dimensões econômicas e geopolíticas do desenvolvimento. Rio de Janeiro: IPEA.

NBSC - National Bureau of Statistics of China (2005, 2008, 2015), http://www.stats.gov.cn/english/statisticaldata/AnnualData, acessadoem 22/01/2016.

NAUGHTON, B. (1996): Growing out of the Plan: Chinese Economic Reform, 19781993. Cambridge: Cambridge University Press.

(2006): "Claiming Profit for the State: SASAC and the Capital Management Budget”. China Leadership Monitor.

(2006): Top-Down Control: SASAC and the Persistence of State

Ownership in China. Paper presented at the conference on "China and the World Economy". Universityof Nottingham.

NONNEMBERG, M. J. (2010): "China: Estabilidade e Crescimento Econômico". Revista de Economia Política, 30, 2 (118): 201-218.

PAULA, L. F. (2014): Sistema Financeiro, Bancos e Financiamento da Economia. Rio de Janeiro: Elsevier.

OLIVEIRA, A. P. (2003): “O salto qualitativo de uma economia continental”. Política Externa, (11) 4: 6-13.

OLIVEIRA, C. A. B (2005): "Reformas econômicas na China". Economia Política Internacional: Análise Estratégica, 05.

RANGEL, I (1952): "Prossegue a recuperação chinesa". Cadernos do Nosso Tempo. 1 (1): 59-64.

: (1981): “A questão financeira”. Revista de Economia Política, (1) 1: pp.

$31-39$.

(1980): Recursos Ociosos e Política Econômica. São Paulo: Hucitec.

REN, D. (2015): "China's Silk Road infrastructure fund explores investment exit strategies". South China Morning Post.

RONG, ZHOU et al (1992): Agriculture in Contemporary China. Beijing: Contemporary China Press.

SING,A. (1993) "The Plan, The Market and Evolutionary Economic Reform in China". UNCTAD: Discussion Papers, 76.

TIEZZI, S. (2015): "Where Is China's Silk Road Actually Going?". The Diplomat. $30 / 03 / 2015$.

WTO - World Trade Organization (2014) - WTO Annual Report https://www.wto.org/english/res_e/publications_e/anrep14_e.htm Acessado a 15/11/2015.

XIAOPING, Deng (1992): "Opening Speech at the Twelfth National Congress of the Communist Party of China". In, XIAOPING, D. (1992): Selected Works. Beijing: Foreign Language Press: 13-17. 
JABBOUR, DANTAS

Recebido em 25 de julho de 2018.

Aceito em 28 de agosto de 2018. 